\title{
Impact of long-term lifestyle programmes on weight loss and cardiovascular risk factors in overweight/obese participants: a systematic review and network meta-analysis
}

\author{
Lukas Schwingshackl $^{1 *}$, Sofia Dias ${ }^{2}$ and Georg Hoffmann ${ }^{1}$
}

\begin{abstract}
Background: The aim of this meta-analysis was to compare the long-term efficacy of diet plus exercise (D $+E)$ vs. diet (D), D + E vs. exercise (E) and D vs. E on anthropometric outcomes and cardiovascular risk factors in overweight and obese participants.

Methods: Electronic searches were performed in MEDLINE and the Cochrane Central Register of controlled trials. Inclusion criteria were as follows: body mass index $\geq 25 \mathrm{~kg} / \mathrm{m}^{2}$ and a minimum intervention period including follow-up of $\geq 12$ months. Outcomes of interest were as follows: anthropometric parameters, blood lipids, blood pressure and cardiorespiratory fitness. Pooled effects were calculated using pairwise random effects and Bayesian random effects network meta-analysis. Results of the corresponding fixed effects models were compared in sensitivity analyses.

Results: Overall, 22 trials (24 reports) met the inclusion criteria and 21 (including 3,521 participants) of them were included in the quantitative analysis. As compared with $D, D+E$ resulted in a significantly more pronounced reduction in body weight [mean differences (MD): $-1.38 \mathrm{~kg}, 95 \%$ confidence interval (Cl) -1.98 to -0.79 ], and fat mass (MD: $-1.65 \mathrm{~kg}, 95 \%$ $\mathrm{Cl}-2.81$ to -0.49$]$, respectively. When comparing $\mathrm{D}+\mathrm{E}$ with $\mathrm{E}, \mathrm{MD}$ in change of body weight $(-4.13 \mathrm{~kg}, 95 \% \mathrm{Cl}-5.62$ to -2.64$)$, waist circumference $(-3.00 \mathrm{~cm}, 95 \% \mathrm{Cl}-5.81$ to -0.20$)$, and fat mass $(-3.60 \mathrm{~kg}, 95 \% \mathrm{Cl}-6.15$ to -1.05$)$ was in favour of combined diet and exercise, respectively. Comparing E vs. D, diet resulted in a significantly more pronounced decrease in body weight (MD: $-2.93 \mathrm{~kg}, 95 \% \mathrm{Cl}-4.18$ to -1.68 ), and fat mass (MD: $-2.20 \mathrm{~kg}, 95 \% \mathrm{Cl}-3.75$ to -0.66 ). $\mathrm{D}+\mathrm{E}$ yielded also the greatest reductions with respect to blood lipids and blood pressure when compared to single applications of $D$ and $E$, respectively. Results from the network meta-analyses confirmed these findings.
\end{abstract}

Conclusions: Moderate-quality evidence from the present network meta-analysis suggests that $D+E$ can be highly recommended for long-term obesity management. Furthermore, the evidence suggests a moderate superiority of D over E with respect to anthropometric outcomes.

Systematic review registration: PROSPERO CRD42013003906

Keywords: Lifestyle, Obesity, Network meta-analysis, Systematic review, Diet, Exercise

\footnotetext{
*Correspondence: lukas.schwingshackl@univie.ac.at

${ }^{1}$ Faculty of Life Sciences, Department of Nutritional Sciences, University of

Vienna, Althanstraße 14 UZA II, A-1090 Vienna, Austria

Full list of author information is available at the end of the article
} 


\section{Background}

In 2008, an estimated 1.4 billion adults were overweight meaning that the prevalence of obesity has more than doubled since 1980. Of these, over 200 million men and nearly 300 million women were obese [1]. Overweight (body mass index (BMI): $\geq 25 \mathrm{~kg} / \mathrm{m}^{2}$ ) and obesity (BMI: $\geq 30 \mathrm{~kg} / \mathrm{m}^{2}$ ) are independent risk factors for non-communicable diseases, especially cardiovascular diseases (CVD) and several types of cancer [2,3]. Exercise and diet are cornerstones in the prevention and management of overweight and obesity. Reductions of fat mass, primarily visceral adipose tissue, are major objectives. Energy expenditure increases with physical activity, especially with aerobic exercise or combined aerobic and resistance training [4,5]. Caloric restriction induces weight loss by negative energy balance. Evidence from meta-analyses indicates that lowcarbohydrate diets have slightly more favourable effects on body weight as compared to low-fat diets. Nevertheless, independent of macronutrient composition, the long-term health effects of diets are as yet unknown, and the observed outcomes appear of little clinical significance [6-9]. Regarding exercise training, results from recent network meta-analyses indicate that combined aerobic and resistance exercise is the most effective training modality in the treatment/prevention of overweight/obesity and type 2 diabetes mellitus $[4,10]$.

Previous meta-analyses by Shaw et al. [11] (including trials: $\geq 3$ months length) as well as Wu et al. [12] ( $\geq 6$ months) focused on intervention trials comparing diet plus exercise $(D+E)$ vs. diet (D) on body weight and BMI as outcome parameters, but anthropometric outcomes such as waist circumference, fat mass, waist to hip ratio and cardiovascular risk factors (blood lipids, blood pressure and cardiorespiratory fitness) were not included. To the best of our knowledge, to date, no metaanalysis has compared the head-to-head and indirect long-term ( $\geq 12$ months) effects of $\mathrm{D}+\mathrm{E}$ vs. D vs. E on anthropometric parameters and cardiovascular risk factors. Therefore, the aim of this study was to conduct a systematic review with pairwise and network meta-analysis of randomized controlled trials to combine the direct and indirect evidence on the efficacy of different lifestyle longterm weight-reducing interventions on anthropometric parameters, blood lipids, blood pressure and cardiorespiratory fitness in participants with a BMI $\geq 25 \mathrm{~kg} / \mathrm{m}^{2}$.

\section{Methods}

The review protocol has been registered in PROSPERO International Prospective Register of Systematic Reviews (crd. york.ac.uk/prospero/index.asp Identifier: CRD42013003906).

\section{Literature search}

Queries of literature were performed using the electronic databases MEDLINE (between 1966 and June 2014) and the Cochrane Trial Register (until June 2014) with no restrictions to language and calendar date using the following search terms: ("ifestyle" OR "exercise" OR "diet") AND ("body weight" OR "lipids") AND ("randomized controlled trial" OR "randomized" OR "clinical trials as topic" OR "placebo" OR "randomly" OR "trial") NOT ("animals" NOT "humans"). Moreover, the reference lists from retrieved articles and systematic reviews and meta-analyses were checked to search for further relevant studies. This systematic review was planned, conducted and reported in adherence to standards of quality for reporting metaanalyses [13]. Literature search was conducted independently by two authors (LS, GH), with disagreements resolved by consensus.

\section{Eligibility criteria}

Studies were included in the meta-analysis if they met all of the following criteria: (i) randomized controlled design; (ii) minimum intervention period including follow-up of 12 months; (iii) body mass index: $\geq 25 \mathrm{~kg} / \mathrm{m}^{2}$; (iv) comparing $\mathrm{D}+\mathrm{E}$ vs. $\mathrm{D}$ or/and $\mathrm{D}+\mathrm{E}$ vs. $\mathrm{E}$ or/and $\mathrm{D}$ vs. E; (v) assessment of "primary outcome" markers: body weight (BW), waist circumference (WC), waist-to-hip ratio (WHR), fat mass (FM) and "secondary outcome" markers: total cholesterol (TC), low-density lipoprotein cholesterol (LDL-C), high-density lipoprotein cholesterol (HDL-C), triacylglycerols (TG), diastolic blood pressure (DBP), systolic blood pressure (SBP) and cardiorespiratory fitness $\left(\mathrm{VO}_{2} \max \right) ;(v i)$ participants with coronary heart disease were excluded; (vii) report post-intervention mean values (if not available change-from-baseline value scores were used) with standard deviation (or basic data to calculate these parameters: standard error or $95 \%$ confidence interval (CI)) according to the Cochrane Handbook [14]; and (viii) $\geq 19$ years of age.

\section{Risk of bias assessment}

Full copies of studies were independently assessed for methodological quality by two authors (LS, GH) using the risk of bias assessment tool by the Cochrane Collaboration. The following sources of bias were detected: selection bias (random sequence generation, allocation concealment), performance/detection bias (blinding of participants and personnel, blinding of outcome assessment), attrition bias (incomplete data outcome) and reporting bias (selective reporting) (Figure 1) [14,15].

\section{Data extraction and statistical analysis}

The following data were extracted from each study: the first author's last name, publication year, study length (including follow-up), participant's sex and age, BMI, sample size,\% T2D, intervention type, characteristics of dietary intervention, characteristics of exercise intervention, dropout rates, post-intervention mean values or change-from-baseline 
value scores with corresponding standard deviation. Data extraction was performed by one author (LS).

Separate pairwise meta-analyses were first used to compare all lifestyle interventions. Network meta-analysis was then used to synthesize all the available evidence [16]. Network meta-analysis methods are extensions of the standard pairwise meta-analysis model which enable simultaneous comparison of multiple interventions whilst preserving the internal randomization of individual trials. They have the advantage of adequately accounting for the correlation in relative effect estimates from three-arm trials as well as providing a single coherent summary of all the evidence.

\section{Pairwise meta-analyses}

For each outcome measure of interest and for each pair of treatments, a random effects inverse variance metaanalysis was performed in order to determine the pooled effect of the intervention in terms of mean differences (MDs) between the post-intervention (or change-frombaseline) values of the different lifestyle interventions [14]. Data were pooled if outcomes were reported by at least three studies. Heterogeneity between trial results was tested with a standard $\chi^{2}$ test. The $I^{2}$ parameter was used to quantify any heterogeneity: $I^{2}=[(Q-d . f)] /$. $100 \%$, where $Q$ is the $\chi^{2}$ statistic and $d . f$. is its degrees of freedom. A value for $I^{2}>50 \%$ was considered to represent substantial heterogeneity [17]. As study characteristics were expected to differ, statistical heterogeneity was also expected and a random effects model was used to estimate MDs with 95\% CIs. In addition, sensitivity analyses were planned to further elucidate the potential influence of heterogeneity due to different study characteristics on the outcome of the pairwise meta-analysis (such as study length, age of participants, risk of bias). Forest plots were generated to illustrate the study-specific effect sizes along with a $95 \% \mathrm{CI}$. To determine the presence of publication bias, we assessed the symmetry of the funnel plots in which mean differences were plotted against their corresponding standard errors taking into account the recommendation by Sterne et al. [18], i.e. that testing for funnel plot asymmetry should only be conducted if the number of studies is ten or larger. Additionally, Begg's and Egger's regression tests were performed to detect small study effects $[19,20]$.

\section{Network meta-analyses}

To account for the expected between-study heterogeneity, random effects network meta-analysis models were used. For each outcome, a common between-study heterogeneity parameter was assumed to reflect the variability between studies of all interventions.

Model fit was assessed by comparing the number of data points to the posterior mean of the residual deviance
Figure 1 Risk of bias assessment tool. Across trials, information is either from trials at a low risk of bias (green), or from trials at unclear risk of bias (yellow), or from trials at high risk of bias (red). 
[16] (these values should be similar in a well-fitting model). Pooled effect sizes from the network meta-analyses are presented as posterior medians and 95\% credible intervals (CrI) (i.e. Bayesian equivalent of confidence intervals) in the appropriate units along with the estimated betweenstudy heterogeneity and its 95\% CrI. Treatments were ranked best, second best and third best based on their efficacy.

To assess sensitivity to the choice of random or fixed effects network meta-analysis models, the two models were compared using the deviance information criteria for each outcome $[16,21]$ which account for both model fit and complexity. The fixed effects model was considered adequate when its deviance information criterion (DIC) was lower than the random effects model (differences $>3$ or 5 are considered meaningful) [16,21]. Mean differences for the fixed effects network meta-analysis (NMA) model are also presented for comparison.

\section{Computation}

For pairwise meta-analyses, data were analysed using the Review Manager 5.1 software, provided by the Cochrane Collaboration (http://ims.cochrane.org/revman). Network meta-analyses were conducted using Markov chain Monte Carlo (MCMC) simulation implemented with the opensource software WinBUGS, version 1.4.3 [22]. The WinBUGS code used is freely available online (program "TSD2-5aRE_Normal_id.odc" for the random effects models and "TSD2-5aFE_Normal_id.odc" for the fixed effects models) [16,23]. Minimally informative normal priors (with mean zero and variance 10,000) were used for all treatment effect parameters, and a uniform $(0,150)$ prior was used for the between-study standard deviation (heterogeneity) parameter. These priors were considered non-informative over the expected range of data. Sensitivity to the prior on the between-study heterogeneity was assessed by varying the upper bound of the uniform distribution, but there was no meaningful change in relative effects or overall conclusions.

Three MCMC chains were used to assess convergence using Brooks-Gelman-Rubin plots and by inspection of the trace plots [24]. Convergence was achieved after 20,000 iterations for all outcomes. Posterior summaries were then obtained from further simulation of 50,000 iterations in each of the three chains (150,000 in total), resulting in a small Monte Carlo error.

Treatments were ranked at each iteration (post-convergence) according to their efficacy, where the "best" treatment was the one with the most favourable outcome (which could be described by a higher or lower $\mathrm{MD}$, depending on the outcome).

The potential for inconsistency was assessed by inspection of the network plots. Where there was a potential for inconsistency, i.e. where there were independent sources of evidence informing direct and indirect estimates, Bayesian $p$ values for the difference between direct and indirect evidence were calculated using the node split method [25,26] implemented in R through GeMTC [27], and direct and indirect estimates were compared.

\section{Results}

In order to ease interpretation of results, the following changes would be considered to be a benefit: $\mathrm{BW}$, decrease; WC, decrease; FM, decrease; WHR, decrease; TC, decrease; LDL-C, decrease; HDL-C, increase; TG, decrease; DBP, decrease; SBP, decrease; $\mathrm{VO}_{2} \max$, increase; altogether, 22 trials (24 reports) met the inclusion criteria and 21 of them were included in the quantitative analysis [28-52]. The detailed steps of the meta-analysis article selection process are given as a flow chart in Figure 2, and full search strategy for PUBMED and the Cochrane Trial Register is given in Additional file 1.

All studies included were randomized controlled trials (RCTs) with a duration ranging between 12 and 72 months, published between 1988 and 2013 and enrolling a total of 3,521 participants, 680 of them being participants with T2D. The mean age varied between 35 and 70 years and the BMI between 25.6 and $38.2 \mathrm{~kg} / \mathrm{m}^{2}$. Seventeen trials compared D+E vs. D, 11 compared D+E vs. E and 14 compared D vs. E. General study characteristics are summarized in Table 1. Regarding the dietary interventions, a major part of the included trials recommended energyreduced low-fat diets ( $\leq 30 \%$ fat of total energy), low in saturated fat, and increased intakes of fruit, vegetables and fibre. Exercise prescription was partly supervised and included aerobic exercise (i.e. jogging, walking, flexibility, circuit training) and resistance training, overall $50 \%-85 \%$ of maximal heart rate.

The direct pairwise and network pooled estimate of effect size for the effects of D $+E$ vs. D, D + E vs. E and D vs. E on anthropometric outcomes, blood lipids, blood pressure and cardiorespiratory fitness are summarized in Table 2.

\section{Anthropometric outcomes/cardiorespiratory fitness Diet + exercise vs. diet}

The weighted mean difference in change of BW [MD: $-1.38 \mathrm{~kg}(95 \% \mathrm{CI}-1.98$ to -0.79$\left.), I^{2}=0 \%\right]$, WC [MD: $-1.68 \mathrm{~cm}$ (95\% CI -2.66 to -0.70$\left.), I^{2}=0 \%\right]$, WHR [MD: $-0.01 \mathrm{U}(95 \% \mathrm{CI}-0.02$ to -0.01$), I^{2}=0 \%$ ] and FM [MD: $-1.65 \mathrm{~kg}(95 \% \mathrm{CI}-2.81$ to -0.49$), I^{2}=61 \%$ ] was significantly more pronounced in the $\mathrm{D}+\mathrm{E}$ group as compared to $\mathrm{D}$, respectively. Furthermore, the $\mathrm{D}+\mathrm{E}$ group revealed significantly more prominent increases in cardiorespiratory fitness (measured as $\mathrm{VO}_{2} \max$ ) [MD: $3.61 \mathrm{ml} / \mathrm{kg} / \mathrm{min}$ (95\% CI 2.07 to 5.14 ), $I^{2}=88 \%$ ] and HDL cholesterol [MD: $1.62 \mathrm{mg} / \mathrm{dl}$ (95\% CI 0.28 to 2.95$), I^{2}=51 \%$ ] as well as decreases in TG [MD: $-10.08 \mathrm{mg} / \mathrm{dl}$ (95\% CI -17.38 


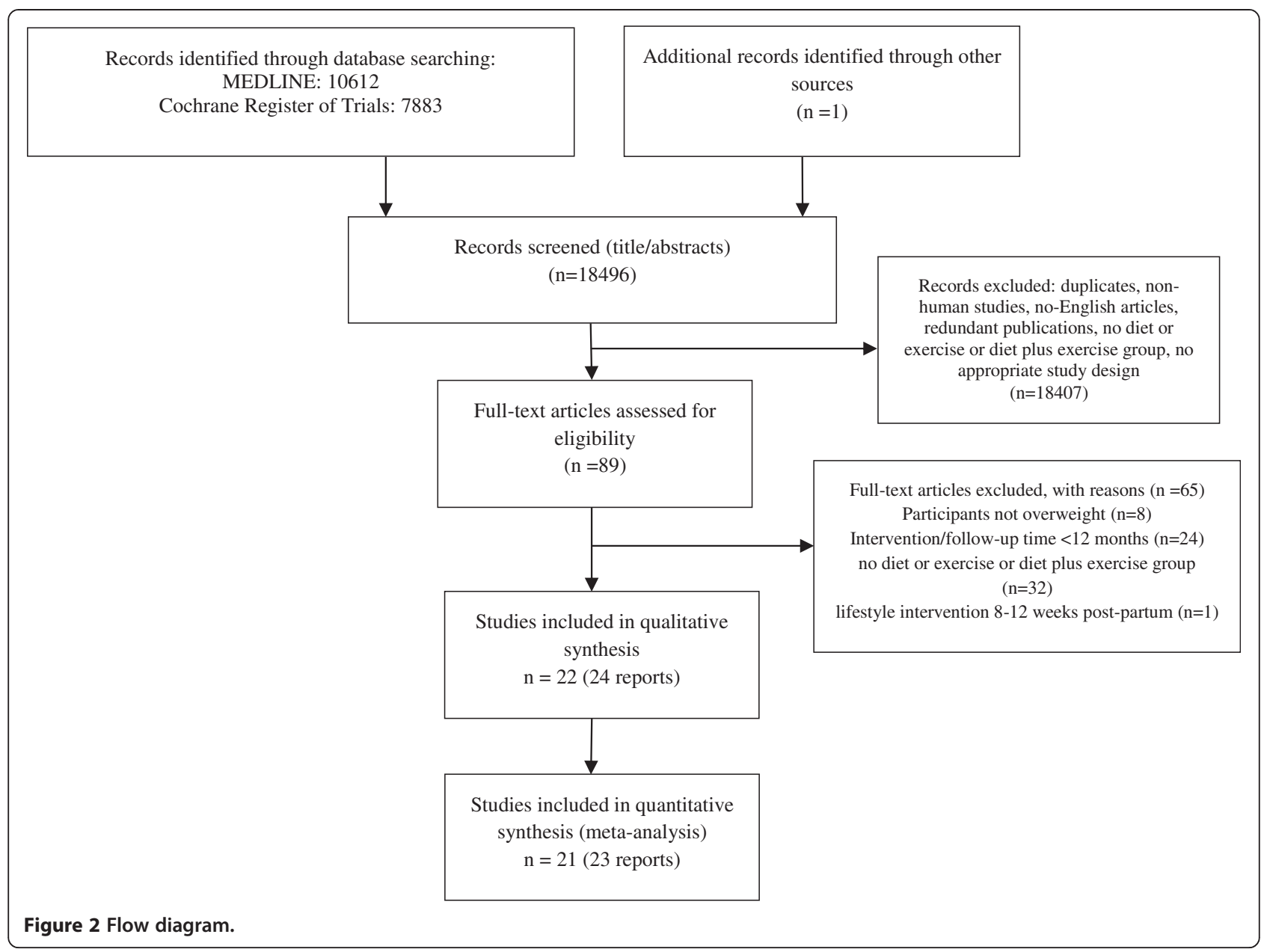

to -2.79$), I^{2}=0 \%$ ] and DBP [MD: $-1.20 \mathrm{mmHg}(95 \%$ CI -2.26 to -0.15$), I^{2}=28 \%$ ]. In contrast, changes observed for TC, LDL-C and SBP did not differ significantly between both groups.

\section{Diet + exercise vs. exercise}

Comparing $\mathrm{D}+\mathrm{E}$ vs. $\mathrm{E}$, a significantly more distinctive reduction in BW [MD: $-4.13 \mathrm{~kg}$ (95\% CI -5.62 to -2.64$)$, $\left.I^{2}=77 \%\right]$, WC [MD: $-3.00 \mathrm{~cm}(95 \% \mathrm{CI}-5.81$ to -0.20$)$, $I^{2}=69 \%$ ], WHR [MD: $-0.01 \mathrm{U}(95 \% \mathrm{CI}-0.02$ to -0.00$), I^{2}=$ 15\%] and FM [MD: $-3.60 \mathrm{~kg}$ (95\% CI -6.15 to -1.05$), I^{2}=$ $92 \%$ ] could be observed in the $\mathrm{D}+\mathrm{E}$ group. Rise of $\mathrm{VO}_{2}$ max was significantly more pronounced in the $\mathrm{D}+\mathrm{E}$ group as well [MD: $2.13 \mathrm{ml} / \mathrm{kg} / \mathrm{min}$ (95\% CI 1.52 to 2.74 ), $I^{2}=9 \%$ ]. With respect to blood lipids, TC [MD: $-11.36 \mathrm{mg} / \mathrm{dl}(95 \%$ CI -15.93 to -6.79$), I^{2}=0 \%$, LDL-C [MD: $-10.03 \mathrm{mg} / \mathrm{dl}$ (95\% CI -14.28 to -5.78$), I^{2}=8 \%$ ], DBP [MD: $-2.06 \mathrm{mmHg}$ (95\% CI -3.39 to -0.72$), I^{2}=0 \%$ ] and SBP [MD: $-2.84 \mathrm{mmHg}(95 \% \mathrm{CI}-4.54$ to -1.13$), I^{2}=0 \%$ ] were reduced more substantially following combined $\mathrm{D}+\mathrm{E}$ when compared to single $\mathrm{E}$ interventions. No significant differences could be observed for HDL-C and TG.

\section{Diet vs. exercise}

Following $\mathrm{D}$ vs. E comparisons, reductions in BW [MD: $-2.93 \mathrm{~kg}$ (95\% CI -4.18 to -1.68$), I^{2}=74 \%$ ], FM [MD: $-2.20 \mathrm{~kg}(95 \% \mathrm{CI}-3.75$ to -0.66$\left.), I^{2}=82 \%\right]$, HDL-C [MD: $-0.96 \mathrm{mg} / \mathrm{dl}$ (95\% CI -1.88 to -0.04$), I^{2}=$ $0 \%$ ] and SBP [MD: $-2.19 \mathrm{mmHg}(95 \% \mathrm{CI}-4.23$ to -0.15$)$, $I^{2}=25 \%$ ] were significantly more pronounced in the D group. WC, WHR, TC, LDL-C and cardiorespiratory fitness were not affected in a different fashion by either D or E.

\section{Network meta-analysis}

Additional file 1: Figure S1 shows the network of included trials. The pooled estimates of effect size for the comparison of $\mathrm{D}+\mathrm{E}$ vs. $\mathrm{D}$ vs. $\mathrm{E}$ using both direct and indirect evidence on anthropometric outcomes, blood lipids, blood pressure and cardiorespiratory fitness are summarized in Table 2.

Both $D+E$ and $D$ were significantly more effective in reducing $\mathrm{BW}$ and $\mathrm{FM}$ when compared to $\mathrm{E}$ alone. $\mathrm{D}+\mathrm{E}$ turned out to be the most effective lifestyle intervention with respect to reduction of BW, WC, FM, TC, LDL-C, TG, DBP, SBP and increasing HDL-C. D + E resulted in 
Table 1 General study characteristics

\begin{tabular}{|c|c|c|c|c|c|c|}
\hline Reference & $\begin{array}{l}\text { Sample size } \\
\text { Baseline BMI }\left(\mathrm{kg} / \mathrm{m}^{2}\right) \\
\% \text { diabetics }\end{array}$ & $\begin{array}{l}\text { Age (years) } \\
\text { Female }(\%)\end{array}$ & $\begin{array}{l}\text { Duration of the active } \\
\text { intervention (follow-up) }\end{array}$ & Study design & $\begin{array}{l}\text { Dietary intervention } \\
\text { Exercise prescription }\end{array}$ & Dropout \\
\hline \multirow{3}{*}{$\begin{array}{l}\text { Anderssen et al. [40] } \\
\text { Reseland et al. [41] }\end{array}$} & 166 & 40 & 12 months & \multirow[t]{3}{*}{$D+E$ vs. D vs. E } & \multirow{3}{*}{$\begin{array}{l}\text { D: increased fish, fruit and vegetables and fibre, reduce } \\
\text { intake of sugar and SFA, no heavy evening meal } \\
\text { E: supervised weekly, aerobic training (strength, flexibility, } \\
\text { circuit training, jogging), } 60 \%-80 \% \text { of peak heart rate }\end{array}$} & \multirow{2}{*}{$\begin{array}{l}\text { D + E: } 3 \% \\
\text { D: } 5 \%\end{array}$} \\
\hline & 28.9 & \multirow[t]{2}{*}{$0 \%$} & \multirow[t]{2}{*}{ (0 months) } & & & \\
\hline & $0 \%$ & & & & & E: 9\% \\
\hline \multirow[t]{3}{*}{ Andrews et al. [42] } & 494 & 60 & 12 months & \multirow[t]{3}{*}{$D+E$ vs. D } & \multirow{3}{*}{$\begin{array}{l}\text { D: aimed at enabling patients to lose } 5 \%-10 \% \text { of their } \\
\text { initial body weight, based on UK dietary guidelines } \\
\text { E: asked to do at least } 30 \text {-min brisk walking on at least } \\
5 \text { days per week }\end{array}$} & \multirow{3}{*}{$\begin{array}{l}D+E: 2 \% \\
D: 1 \%\end{array}$} \\
\hline & 31.55 & $35 \%$ & (0 months) & & & \\
\hline & $100 \%$ & & & & & \\
\hline \multirow[t]{3}{*}{ Borg et al. [43] } & 82 & 42.6 & 8 months & \multirow[t]{3}{*}{$D+E$ vs. D } & D: low-fat diet & \multirow{3}{*}{$\begin{array}{l}\text { D + E: } 18 \% \\
\text { D: } 1 \%\end{array}$} \\
\hline & 32.9 & $0 \%$ & (23 months) & & E: supervised weekly in group, reached $50 \%-60 \%$ of $M H R$, & \\
\hline & n.d. & & & & $\begin{array}{l}\text { included two groups: (1) walking, expended 1,000 kcal } \\
\text { per week, and (2) walking, expended 2,000 kcal per week }\end{array}$ & \\
\hline \multirow[t]{2}{*}{ Brekke et al. [44] } & 49 & $\begin{array}{l}43 \\
33 \%\end{array}$ & 12 months & \multirow[t]{2}{*}{$D+E$ vs. $D$} & $\begin{array}{l}\text { D: Nordic Nutrition Recommendation in addition increase } \\
\text { of low Gl food }\end{array}$ & $D+E: 17 \%$ \\
\hline & $\begin{array}{ll}25.0 \\
100 \%\end{array}$ & & & & $\begin{array}{l}\text { E: the goal to increase physical activity through walking } \\
\text { or other more intensive activities for at least } 30 \mathrm{~min} \text {, four } \\
\text { to five times per week }\end{array}$ & D: $4 \%$ \\
\hline \multirow[t]{3}{*}{ Christensen et al. [52] } & 28 & 63 & 17 months & \multirow[t]{3}{*}{ D vs. E } & \multirow{2}{*}{$\begin{array}{l}\text { D: the goal of the dietary intervention was to produce } \\
\text { and maintain a weight loss of at least } 10 \%\end{array}$} & D: $14 \%$ \\
\hline & 37.05 & 81.3 & (0 months) & & & \multirow[t]{2}{*}{ E: $19 \%$} \\
\hline & $0 \%$ & & & & $\begin{array}{l}\text { E: exercise intervention consisted of a warm-up phase } \\
\text { ( } 10 \mathrm{~min}) \text {, a circuit training phase ( } 45 \mathrm{~min} \text { ) and a cool } \\
\text { down/stretching phase four periods of } 12 \text { weeks and } \\
\text { one period of } 4 \text { weeks (total } 52 \text { weeks). The aim was to } \\
\text { gradually translate the intervention from facility-based } \\
\text { exercises to home-based exercises }\end{array}$ & \\
\hline \multirow[t]{3}{*}{ Fogelholm et al. [45] } & 82 & 35 & 13 months & \multirow[t]{3}{*}{$D+E$ vs. D } & D: low-fat diet & \multirow{3}{*}{$\begin{array}{l}D+E: 2 \% \\
D: 3 \%\end{array}$} \\
\hline & 34 & $100 \%$ & (11 months) & & E: supervised weekly in group, reached $50 \%-60 \%$ of $M H R$, & \\
\hline & $0 \%$ & & & & $\begin{array}{l}\text { included two groups: (1) walking, expended 1,000 kcal per } \\
\text { week, and (2) walking, expend 2,000 kcal per week }\end{array}$ & \\
\hline \multirow[t]{3}{*}{ Foster-Schubert et al. [46] } & 351 & 58 & 12 months & \multirow[t]{3}{*}{$D+E$ vs. D vs. E } & D: total daily energy intake $1,200-2,000 \mathrm{kcal} /$ day on baseline & \multirow{3}{*}{$\begin{array}{l}D+E: 8 \% \\
D: 11 \% \\
E: 9 \%\end{array}$} \\
\hline & 28.6 & $100 \%$ & (0 months) & & 6 months with maintenance thereafter for 12 months & \\
\hline & $0 \%$ & & & & $\begin{array}{l}\text { E: } \geq 45 \text { min of moderate-to-vigorous intensity exercise, } \\
5 \text { days/week ( } 225 \mathrm{~min} / \text { week) for } 12 \text { months; supervised; } \\
70 \%-85 \% \text { of MHR }\end{array}$ & \\
\hline \multirow{4}{*}{$\begin{array}{l}\text { Messier et al. [51] } \\
\text { Nicklas et al. [47] }\end{array}$} & 238 & 68.7 & 18 months & \multirow[t]{4}{*}{$D+E$ vs. D vs. E } & D: the goal of intervention was to produce and maintain & \\
\hline & 34.3 & $73 \%$ & (0 months) & & $\begin{array}{l}\text { an average weight loss of } 5 \% \text { baseline body weight. The } \\
\text { intervention was divided into three phases: intensive }\end{array}$ & D: $23 \%$ \\
\hline & $9 \%$ & & & & transition and maintenance & \\
\hline & & & & & $\begin{array}{l}\text { E: exercise consisted of an aerobic phase, a resistance } \\
\text { training phase, a second aerobic phase and a cooling } \\
\text { down phase }\end{array}$ & \\
\hline
\end{tabular}


Table 1 General study characteristics (Continued)

\begin{tabular}{|c|c|c|c|c|c|c|}
\hline \multirow[t]{4}{*}{ Messier et al. [50] } & 454 & 66 & 18 months & \multirow[t]{4}{*}{$D+E$ vs. D vs. E } & \multirow{3}{*}{$\begin{array}{l}\text { D: diet was based on partial meal replacements, including } \\
\text { up to two meal replacement shakes per day; for the third } \\
\text { meal, participants followed a weekly menu plan and } \\
\text { recipes that were } 500 \text { to } 750 \text { kcal, low in fat and high in } \\
\text { vegetables; initial diet plan provided an energy intake } \\
\text { deficit of } 800 \text { to 1,000 kcal/day }\end{array}$} & $D+E: 11 \%$ \\
\hline & 33.6 & \multirow[t]{3}{*}{$72 \%$} & \multirow[t]{3}{*}{ (0 months) } & & & D: $15 \%$ \\
\hline & $13 \%$ & & & & & E: $11 \%$ \\
\hline & & & & & $\begin{array}{l}\text { E: exercise was conducted for } 1 \mathrm{~h} \text { on } 3 \text { days/week for } \\
18 \text { months; programme consisted of aerobic walking } \\
(15 \mathrm{~min}) \text {, strength training ( } 20 \mathrm{~min}) \text {, a second aerobic } \\
\text { phase ( } 15 \mathrm{~min}) \text { and cool down ( } 10 \mathrm{~min})\end{array}$ & \\
\hline \multirow[t]{3}{*}{ Pan et al. [48] } & 397 & 44.4 & 72 months & \multirow[t]{3}{*}{$D+E$ vs. D vs. E } & \multirow{2}{*}{$\begin{array}{l}\text { D: caloric intake at } 25-30 \mathrm{kcal} / \mathrm{kg} \text { of BW, increased } \\
\text { vegetable intake and reduced intake of sugars, using } \\
\text { individual goals }\end{array}$} & $D+E: 8 \%$ \\
\hline & 25.6 & \multirow[t]{2}{*}{$47 \%$} & \multirow[t]{2}{*}{ (0 months) } & & & D: $8 \%$ \\
\hline & $0 \%, 100 \%$ IGT & & & & $\begin{array}{l}\text { E: increased the amount of exercise at least } 1 \mathrm{U} / \text { day and } \\
\mathrm{U} / \text { day for those less than } 50 \text { years old with no evidence of } \\
\text { heart disease or arthritis. The rate of increase and type of } \\
\text { exercise depending on age, past exercise pattern and } \\
\text { existence for heart problem other than IGT }\end{array}$ & E: $8 \%$ \\
\hline \multirow[t]{3}{*}{ Pritchard et al. [39] } & 39 & 44.25 & 12 months & \multirow[t]{3}{*}{ D vs. E } & D: low-fat diet & D: $0 \%$ \\
\hline & 29.1 & $0 \%$ & (0 months) & & E: aerobic exercise; minimum participation was three & E: $0 \%$ \\
\hline & $0 \%$ & & & & recommended to achieve maximum weight loss & \\
\hline \multirow[t]{3}{*}{ Racette et al. [38] } & 45 & 57.2 & 12 months & \multirow[t]{3}{*}{ D vs. E } & \multirow{2}{*}{$\begin{array}{l}\text { D: decrease energy intake by } 16 \% \text { for the initial } 3 \text { months } \\
\text { and by } 20 \% \text { for the remaining } 9 \text { months; macronutrient } \\
\text { composition was flexible }\end{array}$} & \multirow{3}{*}{$\begin{array}{l}\text { D: } 4 \% \\
\text { E: } 4 \%\end{array}$} \\
\hline & 37.2 & $63 \%$ & (0 months) & & & \\
\hline & $0 \%$ & & & & $\begin{array}{l}\text { E: the goal of the E intervention was to induce an energy } \\
\text { deficit comparable to the } C R \text { intervention by increasing } \\
\text { daily energy expenditure through exercise without } \\
\text { changing caloric intake. Exercise physiologists and trainers } \\
\text { worked with ex-participants individually to establish and } \\
\text { monitor their exercise routines }\end{array}$ & \\
\hline \multirow[t]{3}{*}{ Skender et al. [37] } & 61 & 45 & 12 months & \multirow[t]{3}{*}{$D+E$ vs. D vs. E } & \multirow{2}{*}{$\begin{array}{l}D^{\text {a: }} \text { help your heart eating plan; well-balanced, } \\
\text { low-cholesterol eating plan }\end{array}$} & $D+E: 50 \%$ \\
\hline & 35 & \multirow[t]{2}{*}{$48 \%$} & \multirow[t]{2}{*}{ (12 months) } & & & D: 65\% \\
\hline & $0 \%$ & & & & $\begin{array}{l}\text { E: supervised weekly although group brisk walking at a } \\
\text { level of felt "vigorous" not "strenuous", } 45 \text { min, } \\
4-5 \text { times/week }\end{array}$ & E: 42\% \\
\hline \multirow[t]{3}{*}{ Snel et al. [36] } & 27 & 57.5 & 4 months & \multirow[t]{3}{*}{$D+E$ vs. D } & \multirow{2}{*}{$\begin{array}{l}\text { D: } 4 \text { months: } 450 \mathrm{kcal} / \text { day (consisting of three sachets of } \\
\text { Modifast); weight maintenance: 1,800 kcal }\end{array}$} & \multirow{3}{*}{$\begin{array}{l}D+E: 0 \% \\
D: 0 \%\end{array}$} \\
\hline & 37 & $48 \%$ & (14 months) & & & \\
\hline & $100 \%$ & & & & $\begin{array}{l}\text { E: } 4 \text { days training at home for } 30 \text { min at } 70 \% \text { of maximum } \\
\text { aerobic capacity on a cyclo-ergometer and } 1 \mathrm{~h} \text { in hospital } \\
\text { training under the supervision of a physiotherapist }\end{array}$ & \\
\hline \multirow[t]{3}{*}{ Stefanick et al. [35] } & 276 & $56.9 / 47.8$ & 12 months & \multirow[t]{3}{*}{$D+E$ vs. D vs. E } & D: NCEP step 2 diet & $D+E: 3 \%$ \\
\hline & $26.3 / 27$ & $48 \%$ & (0 months) & & \multirow{2}{*}{$\begin{array}{l}\text { E: supervised weekly, aerobic exercise =16-km jogging } \\
\text { per week }\end{array}$} & D: $3 \%$ \\
\hline & $0 \%$ & & & & & E: 3\% \\
\hline
\end{tabular}


Table 1 General study characteristics (Continued)

\begin{tabular}{|c|c|c|c|c|c|c|}
\hline \multirow[t]{3}{*}{ Villareal et al. [34] } & 80 & 70 & 12 months & \multirow[t]{3}{*}{$D+E$ vs. D vs. E } & \multirow{2}{*}{$\begin{array}{l}\text { D: balance diet with an energy deficit of } 500 \text { to } 750 \mathrm{kcal} \text {. } \\
\text { The diet contained } 1 \mathrm{~g} \text { of high-quality protein/ } / \mathrm{g} \text { of BW } \\
\text { per day }\end{array}$} & $D+E: 11 \%$ \\
\hline & 37 & \multirow[t]{2}{*}{$61 \%$} & \multirow[t]{2}{*}{ (0 months) } & & & D: $15 \%$ \\
\hline & n.d. & & & & $\begin{array}{l}\text { E: three supervised exercise training sessions per week. } \\
\text { Each session was } 90 \mathrm{~min} \text { in duration and consisted of } \\
\text { aerobic and resistance exercise and exercise to improve } \\
\text { flexibility and balance }\end{array}$ & E: 12\% \\
\hline \multirow[t]{3}{*}{ Volpe et al. [33] } & 90 & 44.4 & 6 months & \multirow[t]{3}{*}{$D+E$ vs. D vs. E } & D: intensive (weekly) nutritional classes ( $1-3$ months) & $D+E:$ n.d. \\
\hline & $30.5 / 35.3$ & \multirow[t]{2}{*}{$51 \%$} & \multirow[t]{2}{*}{ (6 months) } & & \multirow{2}{*}{$\begin{array}{l}\text { E: supervised training on Nordic Track } \mathrm{k}^{\mathrm{TM}} \text { indoor skiing } \\
\text { apparatus, 3-4 days per week, } 30 \mathrm{~min} \text { for } 6 \text { weeks }\end{array}$} & D: n.d. \\
\hline & $0 \%$ & & & & & E: n.d. \\
\hline \multirow[t]{3}{*}{ Wadden et al. [32] } & 77 & 42 & 12 months & \multirow[t]{3}{*}{$D+E v s . E$} & D: conventional diet with $1,200-1,500 \mathrm{kcal} /$ day & $D+E: 22 \%$ \\
\hline & 36.5 & \multirow[t]{2}{*}{$100 \%$} & \multirow[t]{2}{*}{ (0 months) } & & \multirow{2}{*}{$\begin{array}{l}\text { E: supervised weekly in a group, } 1 \mathrm{~h} \text {, two times per week, } \\
\text { included in three exercise groups: (1) aerobic, (2) strength } \\
\text { and (3) combined training }\end{array}$} & \multirow[t]{2}{*}{ E: $22 \%$} \\
\hline & $0 \%$ & & & & & \\
\hline \multirow[t]{4}{*}{ Wing et al. [29] } & 114 & 45.5 & 24 months & \multirow[t]{4}{*}{$D+E$ vs. D vs. E } & \multirow{3}{*}{$\begin{array}{l}\text { D: participants were asked to follow an } 800-100 \mathrm{kcal} / \mathrm{day} \\
\text { diet, with } 20 \% \text { of calories as fat, exactly as prescribed for } \\
1-8 \text { weeks of the programme. Gradually more flexible with } \\
\text { calorie goals adjusted to } 1,200-1,500 \mathrm{kcal} / \text { day at week } 16 \text {. } \\
\text { Subject attended weekly group meetings for the first } \\
6 \text { months }\end{array}$} & \multirow{4}{*}{$\begin{array}{l}\text { D }+E: 20 \% \\
\text { D: } 5 \% \\
\text { E: } 16 \%\end{array}$} \\
\hline & 35.9 & \multirow[t]{3}{*}{$79 \%$} & \multirow[t]{3}{*}{ (0 months) } & & & \\
\hline & $0 \%$ & & & & & \\
\hline & & & & & $\begin{array}{l}\text { E: supervised by exercise physiologists weekly in a group. } \\
\text { Mainly brisk walking, } 3 \text { miles, five times per week, total } \\
\text { activity gradually increased to } 1,500 \text { kcal per week }\end{array}$ & \\
\hline \multirow[t]{3}{*}{ Wing et al. [30] } & 30 & 55.56 & 12 months & \multirow[t]{3}{*}{$D+E$ vs. $D$} & \multirow{2}{*}{$\begin{array}{l}\text { D: daily calorie goal designed to produce approximately } \\
1 \mathrm{~kg} / \text { week weight loss. Low-fat diet }\end{array}$} & $D+E: 13 \%$ \\
\hline & 38.2 & \multirow[t]{2}{*}{$70 \%$} & \multirow[t]{2}{*}{ (0 months) } & & & \multirow[t]{2}{*}{ D: $0 \%$} \\
\hline & $100 \%$ & & & & $\begin{array}{l}\text { E: all participants exercised twice a week as a group and } \\
\text { once a week on their own, with each exercise session } \\
\text { lasting approximately } 1 \mathrm{~h}\end{array}$ & \\
\hline \multirow[t]{3}{*}{ Wood et al. [49] } & 152 & $39.1 / 40.3$ & 12 months & \multirow[t]{3}{*}{$D+E$ vs. $D$} & $D^{b}:$ NCEP step 1 diet & $D+E: 14 \%$ \\
\hline & 27.9/30.7 & $48 \%$ & (0 months) & & E: aerobic exercise (brisk walking and jogging) that met & D: $13 \%$ \\
\hline & $0 \%$ & & & & (by the fourth month of the study) & \\
\hline \multirow[t]{3}{*}{ Wood et al. [28] } & 89 & 44.1 & 12 months & \multirow[t]{3}{*}{ D vs. E } & \multirow{2}{*}{$\begin{array}{l}\text { D: individual prescription designed to reduce baseline } \\
\text { total body fat by one third over a 9-month period }\end{array}$} & D: $4 \%$ \\
\hline & n.d & \multirow[t]{2}{*}{$0 \%$} & \multirow[t]{2}{*}{ (0 months) } & & & E: $2 \%$ \\
\hline & $0 \%$ & & & & $\begin{array}{l}\text { E: supervised exercise programme and individual } \\
\text { prescriptions based on estimates of the amount of } \\
\text { energy necessary to decrease total body fat } \\
\text { progressively by one third over } 9 \text { months }\end{array}$ & \\
\hline
\end{tabular}

BMI body mass index, BW body weight, CR caloric restriction, $D$ diet, $E$ exercise, Gl glycaemic index, IGT impaired glucose tolerance, MHR maximal heart rate, NCEP National Cholesterol Education Program, SFA saturated fat, UK United Kingdom; n.d. no data.

${ }^{a}$ Skender et al. [37]: $50 \% \mathrm{CH}, 30 \% \mathrm{~F}, 20 \% \mathrm{P}$, low cholesterol.

${ }^{b}$ Wood et al. [49]: 55\% CH, 30\% F, <10\% SFA, $<300 \mathrm{mg} /$ day. 
Table 2 Estimates (direct pairwise and network meta-analysis, random effects models) of effect size (95\% confidence intervals $/ 95 \%$ credible intervals) expressed as mean difference for the effects of diet + exercise vs. diet, diet + exercise vs. exercise and diet vs. exercise on anthropometric outcomes, blood lipids, blood pressure and cardiorespiratory fitness and between-study heterogeneity variance $\left(\tau^{2} / \tau\right)$

\begin{tabular}{|c|c|c|c|c|c|c|c|c|c|c|}
\hline Outcomes & No. of studies & Sample size & MD & $95 \% \mathrm{Cl}$ & $\tau^{2}$ & $I^{2}$ & MD & $95 \% \mathrm{Crl}$ & $\tau$ & $95 \% \mathrm{Crl}$ \\
\hline \multicolumn{11}{|l|}{$D+E$ vs. $D$} \\
\hline BW (kg) & 17 & 2,317 & -1.38 & {$[-1.98,-0.79]$} & 0.00 & $0 \%$ & -1.38 & {$[-2.62,-0.17]$} & 2.06 & {$[1.37,2.96]$} \\
\hline WC (cm) & 8 & 1,124 & -1.68 & {$[-2.66,-0.70]$} & 0.00 & $0 \%$ & -1.69 & {$[-3.32,-0.20]$} & 1.36 & {$[0.07,3.43]$} \\
\hline FM (kg) & 9 & 1,012 & -1.65 & {$[-2.81,-0.49]$} & 1.95 & $61 \%$ & -1.89 & {$[-3.44,-0.43]$} & 2.08 & {$[1.29,3.24]$} \\
\hline WHR (U) & 6 & 646 & -0.01 & {$[-0.02,-0.01]$} & 0.00 & $0 \%$ & -0.01 & {$[-0.05,0.03]$} & 0.06 & {$[0.04,0.10]$} \\
\hline $\mathrm{TC}(\mathrm{mg} / \mathrm{dl})$ & 9 & 1,175 & -2.19 & {$[-7.84,3.46]$} & 54.09 & $62 \%$ & -2.51 & {$[-7.61,2.29]$} & 5.42 & {$[0.86,10.84]$} \\
\hline LDL-C (mg/dl) & 8 & 1,147 & -0.93 & {$[-6.14,4.27]$} & 45.03 & $65 \%$ & -1.54 & {$[-6.16,3.14]$} & 5.29 & {$[1.26,9.97]$} \\
\hline $\mathrm{HDL}-\mathrm{C}(\mathrm{mg} / \mathrm{dl})$ & 9 & 1,175 & 1.62 & {$[0.28,2.95]$} & 2.46 & $51 \%$ & -1.29 & {$[-1.38,3.86]$} & 4.03 & {$[2.38,6.33]$} \\
\hline $\mathrm{TG}(\mathrm{mg} / \mathrm{dl})$ & 9 & 1,175 & -10.08 & {$[-17.38,-2.79]$} & 0.00 & $0 \%$ & -9.90 & {$[-19.98,-0.96]$} & 7.28 & {$[0.37,19.4]$} \\
\hline $\mathrm{DBP}(\mathrm{mmHg})$ & 7 & 1,099 & -1.20 & {$[-2.26,-0.15]$} & 0.74 & $28 \%$ & -1.10 & {$[-2.34,0.01]$} & 0.92 & {$[0.04,2.45]$} \\
\hline $\mathrm{SBP}(\mathrm{mmHg})$ & 7 & 1,099 & -0.24 & {$[-1.45,0.97]$} & 0.00 & $0 \%$ & -0.39 & {$[-1.89,1.01]$} & 0.87 & {$[0.04,2.62]$} \\
\hline $\mathrm{VO}_{2} \max (\mathrm{ml} / \mathrm{kg} / \mathrm{min})$ & 6 & 810 & 3.61 & {$[2.07,5.14]$} & 4.19 & $88 \%$ & 3.75 & {$[2.28,5.32]$} & 1.94 & {$[1.13,3.18]$} \\
\hline \multicolumn{11}{|l|}{$\mathrm{D}+\mathrm{E}$ vs. $\mathrm{E}$} \\
\hline BW (kg) & 9 & 1,350 & -4.13 & {$[-5.62,-2.64]$} & 4.36 & $77 \%$ & -4.32 & {$[-5.74,-2.90]$} & 2.06 & {$[1.37,2.96]$} \\
\hline WC (cm) & 3 & 409 & -3.00 & {$[-5.81,-0.20]$} & 5.24 & $69 \%$ & -3.45 & {$[-5.32,-1.23]$} & 1.36 & {$[0.07,3.43]$} \\
\hline FM (kg) & 5 & 690 & -3.60 & {$[-6.15,-1.05]$} & 8.82 & $92 \%$ & -3.87 & {$[-5.61,-2.18]$} & 2.08 & {$[1.29,3.24]$} \\
\hline WHR (U) & 4 & 420 & -0.01 & {$[-0.02,-0.00]$} & 0.00 & $15 \%$ & -0.007 & {$[-0.06,0.04]$} & 0.06 & {$[0.04,0.10]$} \\
\hline $\mathrm{TC}(\mathrm{mg} / \mathrm{dl})$ & 4 & 420 & -11.36 & {$[-15.93,-6.79]$} & 0.00 & $0 \%$ & -7.50 & {$[-13.47,-1.39]$} & 5.42 & {$[0.86,10.84]$} \\
\hline LDL-C (mg/dl) & 4 & 420 & -10.03 & {$[-14.28,-5.78]$} & 2.22 & $8 \%$ & -5.90 & {$[-11.39,-0.23]$} & 5.29 & {$[1.26,9.97]$} \\
\hline HDL-C (mg/dl) & 4 & 420 & -0.34 & {$[-2.82,2.14]$} & 6.80 & $76 \%$ & 0.17 & {$[-3.14,3.32]$} & 4.03 & {$[2.38,6.33]$} \\
\hline $\mathrm{TG}(\mathrm{mg} / \mathrm{dl})$ & 4 & 420 & -11.18 & {$[-26.99,4.62]$} & 138.3 & $38 \%$ & -13.34 & {$[-25.92,-2.12]$} & 7.28 & {$[0.37,19.4]$} \\
\hline $\mathrm{DBP}(\mathrm{mmHg})$ & 4 & 420 & -2.06 & {$[-3.39,-0.72]$} & 0.00 & $0 \%$ & -2.22 & {$[-3.93,-0.74]$} & 0.92 & {$[0.04,2.45]$} \\
\hline $\mathrm{SBP}(\mathrm{mmHg})$ & 4 & 420 & -2.84 & {$[-4.54,-1.13]$} & 0.00 & $0 \%$ & -2.70 & {$[-4.57,-0.85]$} & 0.87 & {$[0.04,2.62]$} \\
\hline $\mathrm{VO}_{2} \max (\mathrm{ml} / \mathrm{kg} / \mathrm{min})$ & 5 & 645 & 2.13 & {$[1.52,2.74]$} & 0.05 & $9 \%$ & 2.24 & {$[0.57,3.90]$} & 1.94 & {$[1.13,3.18]$} \\
\hline \multicolumn{11}{|l|}{ D vs. E } \\
\hline BW (kg) & 13 & 1,638 & -2.93 & {$[-4.18,-1.68]$} & 3.70 & $73 \%$ & -2.93 & {$[-4.20,-1.66]$} & 2.06 & {$[1.37,2.96]$} \\
\hline WC (cm) & 4 & 539 & -1.75 & {$[-4.12,0.62]$} & 4.80 & $71 \%$ & -1.76 & {$[-3.48,0.44]$} & 1.36 & {$[0.07,3.43]$} \\
\hline FM (kg) & 9 & 964 & -2.20 & {$[-3.75,-0.66]$} & 4.66 & $82 \%$ & -1.97 & {$[-3.45,-0.45]$} & 2.08 & {$[1.29,3.24]$} \\
\hline WHR (U) & 4 & 414 & -0.00 & {$[-0.01,0.01]$} & 0.00 & $16 \%$ & 0.002 & {$[-0.05,0.05]$} & 0.06 & {$[0.04,0.10]$} \\
\hline $\mathrm{TC}(\mathrm{mg} / \mathrm{dl})$ & 7 & 665 & -3.91 & {$[-8.11,0.30]$} & 9.04 & $22 \%$ & -4.98 & {$[-10.22,0.64]$} & 5.42 & {$[0.86,10.84]$} \\
\hline LDL-C (mg/dl) & 7 & 665 & -3.19 & {$[-6.85,0.48]$} & 6.45 & $21 \%$ & -4.36 & {$[-9.25,0.70]$} & 5.29 & {$[1.26,9.97]$} \\
\hline HDL-C (mg/dl) & 7 & 665 & -0.96 & {$[-1.88,-0.04]$} & 0.00 & $0 \%$ & -1.12 & {$[-4.06,-1.76]$} & 4.03 & {$[2.38,6.33]$} \\
\hline TG (mg/dl) & 7 & 665 & -3.80 & {$[-12.21,4.62]$} & 0.00 & $0 \%$ & -3.44 & {$[-13.99,6.65]$} & 7.28 & {$[0.37,19.4]$} \\
\hline $\mathrm{DBP}(\mathrm{mmHg})$ & 6 & 573 & -1.33 & {$[-3.00,0.35]$} & 11.19 & $37 \%$ & -1.12 & {$[-2.67,0.31]$} & 0.92 & {$[0.04,2.45]$} \\
\hline $\mathrm{SBP}(\mathrm{mmHg})$ & 6 & 578 & -2.19 & {$[-4.23,-0.15]$} & 2.07 & $25 \%$ & -2.31 & {$[-4.10,0.51]$} & 0.87 & {$[0.04,2.62]$} \\
\hline $\mathrm{VO}_{2} \max (\mathrm{ml} / \mathrm{kg} / \mathrm{min})$ & 6 & 677 & -1.16 & {$[-2.42,0.09]$} & 2.17 & $80 \%$ & -1.15 & {$[-3.16,0.04]$} & 1.94 & {$[1.13,3.18]$} \\
\hline
\end{tabular}

$B W$ body weight, $C l$ confidence intervals, $D$ diet, $D B P$ diastolic blood pressure, $D+E$ diet and exercise, $E$ exercise, $F M$ fat mass, $H D L-C$ high-density lipoprotein cholesterol, $L D L-C$ low-density lipoprotein cholesterol, SBP systolic blood pressure, $T C$ total cholesterol, $T G$ triacyglycerols, $V O_{2}$ max maximal oxygen uptake, WC waist circumference, WHR waist-to-hip ratio.

a high $(>75 \%)$ probability to be the best for most outcomes. There is greater uncertainty regarding which treatment is the best for HDL-C, although again $\mathrm{D}+\mathrm{E}$ yielded the highest probability of being the best. $\mathrm{D}$ turned out be the second effective lifestyle intervention for BW, WC, FM, TC and DBP ( $>75 \%$ probability).

There was potential for inconsistency in the networks for all outcomes except WHR (Additional file 1: Figure S1). 
There was some evidence of inconsistency for the outcome LDC ( $p$ value $=0.02$ ) although this might be due to chance since several $p$ values for inconsistency are being calculated (Additional file 1: Table S8). After inspection of the evidence on this outcome we did not identify a reason for this apparent inconsistency.

\section{Risk of bias}

The dropout rates ranged from $0 \%$ to $65 \%$, with 11 out of 22 trials reporting dropout rates $<10 \%$ (Table 1 ). Pairwise meta-analysis resulted in no significant dropout differences $(p=0.37)$ between $\mathrm{D}+\mathrm{E}(122 / 1,257)$ vs. $\mathrm{D}(111 / 1,152), \mathrm{D}+\mathrm{E}(79 / 714)$ vs. $\mathrm{D}(82 / 715)(p=0.96)$ and $\mathrm{D}+\mathrm{E}(87 / 701)$ vs. $\mathrm{D}(84 / 706)(p=0.34)$. Eight trials (nine reports) reported random sequence generation $[34,35,42,43,46,47,50-52]$, and only two trials reported allocation concealment $[42,52]$. None of the studies reported blinding of volunteers towards mode of intervention (not possible in diet/exercise trials), and four trials (five reports) appear to have adequate blinding of outcome assessment $[46,47,50-52]$. High risk of bias was defined as fewer than three out of a maximum yield of six low risk of bias items using the risk of bias assessment tool from the Cochrane Collaboration. Only seven low risk of bias trials (eight reports) were identified [34,35,42,46,50-52], and sensitivity analyses were performed for studies with a low risk of bias (Additional file 1: Table S1).

\section{Sensitivity analysis}

Sensitivity analyses (pairwise meta-analysis) were performed for obese, study length $\geq 24$ months, older participants (age: $\geq 50$ years) and low risk of bias studies. Comparison of long-term vs. short-term trials resulted in smaller reductions in body weight (Additional file 1: Tables S2-S3). The results of the primary analysis $(\mathrm{D}+\mathrm{E}$ vs. D and D vs. E) could be confirmed including only obese participants (Additional file 1: Table S4), except for $\mathrm{WC}$ and $\mathrm{VO}_{2}$ max. Including only participants $\geq 50$ years of age (Additional file 1: Table S5), results of the primary analysis could not be confirmed regarding anthropometric outcomes when comparing D + E vs. D (only four to eight trials available), whilst the results of the main analysis for D vs. E were not affected. Sensitivity analyses excluding trials with a high risk of bias changed some summary estimates (D+E vs. D) for anthropometric outcomes and became statistically non-significant (with the exception of D vs. E comparisons).

\section{Fixed vs. random effects models}

Results of the pairwise meta-analyses with a fixed effects model are presented in Additional file 1: Table S6. Overall, the results of the main analysis could be confirmed in the fixed pairwise sensitivity analyses. However comparing D vs. E resulted in a significant reduction in WC,
TC and LDL-C in the fixed effects model, which was not observed in the random effects model, that were more pronounced in the D group. However, we note that the between-study heterogeneity was moderate to high for these outcomes and therefore the fixed effects model may not be appropriate.

In the NMA, comparing the DIC for fixed and random effects models suggests that the fixed effects model might be appropriate for DBP, SBP, TC, TG and WC (Additional file 1: Table S7). The estimated treatment effects using fixed effects network meta-analyses are given in Additional file 1: Table S6. Some differences could be observed between the random and fixed effects models. In the fixed effects model, comparing $\mathrm{D}$ vs. $\mathrm{E}$ resulted in a significant reduction in WC, TC, LDL-C, SBP and $\mathrm{VO}_{2}$ max, favouring $\mathrm{D}$ (with the exception of $\mathrm{VO}_{2} \max$ ), compared to the random effects model. Furthermore, the fixed effects model showed a significant reduction in WHR, comparing $\mathrm{D}+\mathrm{E}$ vs. $\mathrm{D} / \mathrm{E}$, and increase in HDL-C compared to E. Again, we note that the fixed effects model was not always considered suitable (Additional file 1: Table S7).

\section{Publication bias}

Begg's and Egger's regression tests provided no evidence of substantial publication bias (data not shown). Funnel plots were generated only if specific outcome measures were provided by at least ten different trials (some trials provide more than one data point for analysis as they report results for subgroups: men and women) [18]. The plot with respect to effect size change for body weight in response to $\mathrm{D}+\mathrm{E}$ vs. $\mathrm{D}$ vs. $\mathrm{E}$ indicates little asymmetry (Additional file 1: Figures S2-S3). Thus, publication bias cannot be completely excluded as a factor affecting the results of the present meta-analysis.

\section{Discussion}

The primary findings of this systematic review and metaanalysis of long-term trials are that a combination of $\mathrm{D}+\mathrm{E}$ is more effective in reducing BW compared to either D or $\mathrm{E}$ in overweight and obese participants. Furthermore, improvements in WC, WHR and FM were significantly more pronounced following $\mathrm{D}+\mathrm{E}$ compared to either $\mathrm{D}$ or $\mathrm{E}$. The combination of $\mathrm{D}+\mathrm{E}$ is also the most powerful lifestyle intervention to reduce blood lipids and blood pressure. Compared to E, a dietary intervention resulted in significantly greater reductions in body weight, fat mass and systolic blood pressure. Pooling both direct and indirect evidence on $D+E, D$ and $E$ via network meta-analysis demonstrated that $\mathrm{D}+\mathrm{E}$ was the most efficacious exercise intervention regarding its impact on $\mathrm{BW}, \mathrm{WC}, \mathrm{FM}, \mathrm{TC}$, LDL-C, TG, DBP and $\mathrm{VO}_{2}$ max.

The findings of this meta-analysis, i.e. diet being significantly more effective in reducing BW than exercise, are supported by results of a 2006 Cochrane review [11] 
and are consistent with results of another meta-analysis [53]. Therefore, caloric restriction appears the most powerful method for achieving weight loss in overweight and obese people. The amount of weight loss yielded by diet and exercise can be compared to the corresponding results of the most effective pharmacological interventions such as orlistat $(4.12 / 3.1 \mathrm{~kg}$ at 12 months) [54,55]. A recent systematic review concluded that orlistat, lorcaserin and phentermine/topiramate ER when used as an adjunct to lifestyle intervention, could induce a clinically relevant $(\geq 5 \%)$ 12-month weight loss [56]. The interpretation of our network meta-analysis is restricted by the fact that none of the trials evaluated the impact of their interventions on clinical outcomes. It should be noted, however, that no anti-obesity drugs have been shown to have a favourable effect on CVD morbidity and mortality as well [57]. Furthermore anti-obesity drugs were associated with increased risk of total adverse events, tachycardia, gastrointestinal disease, hypertension and mouth dryness [58].

Previous studies reported that a $5 \%$ reduction of $\mathrm{BW}$ is associated with a reduced risk of T2D incidence and other metabolic disorders. A 5-kg weight loss over time could account for a $55 \%$ reduction in the risk of diabetes over the mean of 3.2 years of follow-up in a high-risk population [59]. Regarding visceral adipose tissue, a comprehensive meta-analysis of cohort studies showed that a $1-\mathrm{cm}$ increase in WC and a $0.01-\mathrm{U}$ increase in WHR are associated with a $2 \%-5 \%$ increase in risk of future CVD [60]. Applying this data to the results of the present meta-analysis, $\mathrm{D}+\mathrm{E}$ would be associated with a CVD risk reduction of $\sim 3 \%-6 \%$. Improvements on anthropometric outcomes were more distinct in younger participants compared with older which might be explained by the fact that younger participants were able to perform more intense exercise sessions. However, since not all trials applied supervised exercise, no definitive explanation can be given.

With respect to blood lipids, a meta-analysis of 70 studies indicate that each kilogram of weight loss was associated with a $1.9 \mathrm{mg} / \mathrm{dl}$ decrease in TC and a $0.77 \mathrm{mg} / \mathrm{dl}$ decrease in LDL-C, respectively [61]. Furthermore, RCTs showed that especially aerobic exercise was associated with an increase in HDL-C [62]. These associations could be confirmed in the present meta-analysis.

The predominant dietary intervention implemented in the included trials was either an energy-restricted lowfat diet or an energy-balanced moderate-fat diet. In general, the composition of diets was approximately at least $500 \mathrm{kcal}$ below the estimated energy need, and fat intake was $\leq 30 \%$ of total energy content. In the $\mathrm{D}+\mathrm{E}$ and $\mathrm{D}$ trials, 1,200 kcal for women and 1,500 kcal for men were generally prescribed. In the dietary intervention trials, general guidelines for physical activity were recommended.
However, in the D + E trials, specific goals for physical activity/exercise were implemented. Network meta-analyses provides evidence that a combination of aerobic and resistance training should be recommended in the prevention and treatment of overweight, obesity and associated diseases $[4,10]$.

Cardiorespiratory fitness is associated with cardiovascular mortality and cancer in men and women $[63,64]$. A pooled analysis investigating the effects of cardiorespiratory fitness on all-cause mortality and cardiovascular events demonstrated that a 1-unit increase in metabolic equivalents was associated with a $13 \%$ and $15 \%$ reduction in risk of all-cause mortality and coronary heart disease (CHD)/CVD, respectively [65]. Transferring this data to the results of the present meta-analysis, D + E reduced the risk of all-cause mortality by $14 \%$ and of CVD/CHD by $16 \%$ and approximately $8 \%$ (mortality) and 9\% (CVD/CHD) following applications of single lifestyle interventions, respectively. A possible explanation of the superior effect of D $+E$ on cardiorespiratory fitness could be the greater weight loss induced by caloric restriction.

The present systematic review has several limitations. A major limitation is that no search for unpublished studies and any additional data sources and strategies (author contacts, trial registers) was performed. Another limitation is the fact that not all potential effect modifiers were accounted for. Often participants in different arms of trials did not receive equal numbers of contacts. It could be argued that contact time rather than the specific elements of the intervention affected participant's weight and cardiovascular risk factor outcomes. Heterogeneity could be observed for some outcome parameters, probably introduced by differences between trials, including different $\mathrm{D}+\mathrm{E}$ regimens. Publication bias cannot be excluded to affect the results on any meta-analysis; however, formal statistical testing did not suggest publication bias for the current analysis. Had there been evidence of publication bias and if more studies were available, regression techniques could be used to adjust for this [66].

Although many studies included in our analysis had a substantial dropout rate (see Table 1), intention-to-treat analyses were generally not conducted. However, dropouts were generally similar for all intervention groups. Taken together, $2 / 3$ of the included trials were judged as being at high risk of bias. Therefore, the results should be interpreted in a conservative manner. Strengths of this research include the application of the network meta-analysis, as well as the fact that there was no evidence of inconsistency for most outcomes, and an overall sample size of 3,521 participants.

\section{Conclusions}

The present network meta-analysis provides moderatequality evidence that $\mathrm{D}+\mathrm{E}$ induces moderate long-term 
weight loss and reduces blood lipids and blood pressure when compared to $\mathrm{E}$ or $\mathrm{D}$ as single interventional measures, respectively. In addition, the evidence suggests moderate superiority of D over E regarding anthropometric outcome parameters. The current findings seem to be clinically relevant for public health, in particular for encouraging a combination of diet and exercise for primary prevention of overweight and obesity. Future trials should investigate the long-term effects of different training modalities (aerobic, resistance or combined training) in combination with dietary interventions for the prevention and treatment of overweight and obesity.

\section{Additional file}

\section{Additional file 1: Full search strategy: PUBMED. Table S1}

sensitivity analysis (low risk of bias). Table S2: sensitivity analysis (study length: $\geq 24$ months). Table S3: sensitivity analysis (study length, $<24$ months). Table S4: sensitivity analysis (obese participants). Table S5: sensitivity analysis (participants $\geq 50$ years of age). Table S6: sensitivity analyses (direct pairwise and network meta-analysis, fixed effects models). Table S7: model fit statistics for the random and fixed effects network meta-analysis models. Table S8: estimates of the effects of diet + exercise vs. exercise from direct and indirect evidence in node split models. Figure S1: network graph. Figure S2: funnel plot for body weight (diet + exercise vs. diet). Figure S3: funnel plot for body weight (diet + exercise vs. exercise). Figure S4: funnel plot for body weight (diet vs. exercise).

\section{Competing interests}

The authors declare that they have no competing interests.

\section{Authors' contributions}

LS extracted the data. LS, SD and GH conducted the data analysis, interpreted the results and drafted and finalized the manuscript. All authors read and approved the final manuscript.

\section{Author details}

${ }^{1}$ Faculty of Life Sciences, Department of Nutritional Sciences, University of Vienna, Althanstraße 14 UZA II, A-1090 Vienna, Austria. ${ }^{2}$ School of Social \& Community Medicine, University of Bristol, Canynge Hall, 39 Whatley Road, Bristol BS8 2PS, UK

Received: 22 July 2014 Accepted: 20 October 2014

Published: 30 October 2014

\section{References}

1. WHO: Obesity and Overweight. Fact sheet No. 311. 2011, [http://www. who.int/mediacentre/factsheets/fs311/en/]

2. Guh DP, Zhang W, Bansback N, Amarsi Z, Birmingham CL, Anis AH: The incidence of co-morbidities related to obesity and overweight: a systematic review and meta-analysis. BMC Public Health 2009, 9:88.

3. Renehan AG, Tyson M, Egger M, Heller RF, Zwahlen M: Body-mass index and incidence of cancer: a systematic review and meta-analysis of prospective observational studies. Lancet 2008, 371(9612):569-578.

4. Schwingshackl L, Dias S, Strasser B, Hoffmann G: Impact of different training modalities on anthropometric and metabolic characteristics in overweight/obese subjects: a systematic review and network meta-analysis. PLoS One 2013, 8(12):e82853.

5. Andersen RE, Wadden TA, Bartlett SJ, Zemel B, Verde TJ, Franckowiak SC: Effects of lifestyle activity vs structured aerobic exercise in obese women: a randomized trial. JAMA 1999, 281(4):335-340.

6. Bueno NB, de Melo IS, de Oliveira SL, da Rocha Ataide T: Very-low-carbohydrate ketogenic diet v. low-fat diet for long-term weight loss: a meta-analysis of randomised controlled trials. Br J Nutr 2013, 110(7):1178-1187.
7. SchwingshackI L, Hoffmann G: Comparison of effects of long-term low-fat vs high-fat diets on blood lipid levels in overweight or obese patients: a systematic review and meta-analysis. J Acad Nutr Diet 2013, 113(12):1640-1661.

8. Santos FL, Esteves SS, da Costa PA, Yancy WS, Nunes JP: Systematic review and meta-analysis of clinical trials of the effects of low carbohydrate diets on cardiovascular risk factors. Obes Rev 2012, 13(11):1048-1066.

9. Schwingshackl L, Hoffmann G: Comparison of the long-term effects of high-fat v. low-fat diet consumption on cardiometabolic risk factors in subjects with abnormal glucose metabolism: a systematic review and meta-analysis. Br J Nutr 2014, 111(12):2047-2058.

10. Schwingshackl L, Missbach B, Dias S, Konig J, Hoffmann G: Impact of different training modalities on glycaemic control and blood lipids in patients with type 2 diabetes: a systematic review and network meta-analysis. Diabetologia 2014, 57(9):1789-1797.

11. Shaw K, Gennat H, O'Rourke P, Del Mar C: Exercise for overweight or obesity. Cochrane Database Syst Rev 2006, 4, CD003817.

12. Wu T, Gao X, Chen M, van Dam RM: Long-term effectiveness of diet-plusexercise interventions vs. diet-only interventions for weight loss: a meta-analysis. Obes Rev 2009, 10(3):313-323.

13. Moher D, Liberati A, Tetzlaff J, Altman DG: Preferred reporting items for systematic reviews and meta-analyses: the PRISMA statement. PLoS Med 2009, 6(7):e1000097.

14. Higgins JP, Green S: Cochrane Handbook of Systematic Reviews, Version 5.1.0, The Cochrane Collaboration.

15. Higgins JP, Altman DG, Gotzsche PC, Juni P, Moher D, Oxman AD, Savovic J, Schulz KF, Weeks L, Sterne JA: The Cochrane Collaboration's tool for assessing risk of bias in randomised trials. BMJ 2011, 343:d5928.

16. Dias S, Sutton AJ, Ades AE, Welton NJ: Evidence synthesis for decision making 2: a generalized linear modeling framework for pairwise and network metaanalysis of randomized controlled trials. Med Decis Making 2013, 33(5):607-617.

17. Higgins JP, Thompson SG, Deeks JJ, Altman DG: Measuring inconsistency in meta-analyses. BMJ 2003, 327(7414):557-560.

18. Sterne JA, Sutton AJ, loannidis JP, Terrin N, Jones DR, Lau J, Carpenter J, Rucker G, Harbord RM, Schmid CH, Tetzlaff J, Deeks JJ, Peters J, Macaskill P, Schwarzer G, Duval S, Altman DG, Moher D, Higgins JP: Recommendations for examining and interpreting funnel plot asymmetry in meta-analyses of randomised controlled trials. BMJ 2011, 343:d4002.

19. Begg $C B$, Mazumdar $\mathrm{M}$ : Operating characteristics of a rank correlation test for publication bias. Biometrics 1994, 50(4):1088-1101.

20. Egger M, Davey Smith G, Schneider M, Minder C: Bias in meta-analysis detected by a simple, graphical test. BMJ 1997, 315(7109):629-634.

21. Spiegelhalter DJ, Best NG, Carlin BP, van der Linde A: Bayesian measures of model complexity and fit. J R Stat Soc (B) 2002, 64:583-616.

22. Lunn DJ, Thomas A, Best N, Spiegelhalter D: WinBUGS - a Bayesian modelling framework: concepts, structure, and extensibility. Stat Comput 2000, 10:325-337.

23. Dias S, Welton NJ, Sutton AJ, Ades AE: NICE DSU technical support document 2: a generalised linear modelling framework for pairwise and network meta-analysis of randomised controlled trials. 2011, [http://uww.nicedsu.org.uk]

24. Brooks SP, Gelman A: Alternative methods for monitoring convergence of iterative simulations. J Comput Graphical Stat 1998, 7:434-455.

25. Dias S, Welton NJ, Caldwell DM, Ades AE: Checking consistency in mixed treatment comparison meta-analysis. Stat Med 2010, 29(7-8):932-944.

26. Dias S, Welton NJ, Sutton AJ, Caldwell DM, Lu G, Ades AE: Evidence synthesis for decision making 4: inconsistency in networks of evidence based on randomized controlled trials. Med Decis Making 2013, 33(5):641-656.

27. van Valkenhoef $G, L u G$, de Brock B, Hillege $H$, Ades $A E$, Welton NJ: Automating network meta-analysis. Res Synth Method 2012, 3:285-299.

28. Wood PD, Stefanick ML, Dreon DM, Frey-Hewitt B, Garay SC, Williams PT, Superko HR, Fortmann SP, Albers JJ, Vranizan KM, Ellsworth NM, Terry RB, Haskell WL: Changes in plasma lipids and lipoproteins in overweight men during weight loss through dieting as compared with exercise. $N$ Engl J Med 1988, 319(18):1173-1179.

29. Wing RR, Venditti E, Jakicic JM, Polley BA, Lang W: Lifestyle intervention in overweight individuals with a family history of diabetes. Diabetes Care 1998, 21(3):350-359.

30. Wing RR, Epstein LH, Paternostro-Bayles M, Kriska A, Nowalk MP, Gooding W: Exercise in a behavioural weight control programme for obese patients with type 2 (non-insulin-dependent) diabetes. Diabetologia 1988, 31(12):902-909.

31. Williams PT, Krauss RM, Stefanick ML, Vranizan KM, Wood PD: Effects of low-fat diet, calorie restriction, and running on lipoprotein subfraction 
concentrations in moderately overweight men. Metabolism 1994, 43(5):655-663.

32. Wadden TA, Vogt RA, Foster GD, Anderson DA: Exercise and the maintenance of weight loss: 1-year follow-up of a controlled clinical trial. J Consult Clin Psychol 1998, 66(2):429-433.

33. Volpe SL, Kobusingye H, Bailur S, Stanek E: Effect of diet and exercise on body composition, energy intake and leptin levels in overweight women and men. J Am Coll Nutr 2008, 27(2):195-208.

34. Villareal DT, Chode S, Parimi N, Sinacore DR, Hilton T, Armamento-Villareal R, Napoli N, Qualls C, Shah K: Weight loss, exercise, or both and physical function in obese older adults. N Engl J Med 2011, 364(13):1218-1229.

35. Stefanick ML, Mackey S, Sheehan M, Ellsworth N, Haskell WL, Wood PD: Effects of diet and exercise in men and postmenopausal women with low levels of HDL cholesterol and high levels of LDL cholesterol. N Engl J Med 1998, 339(1):12-20.

36. Snel M, van Diepen JA, Stijnen T, Pijl H, Romijn JA, Meinders AE, Voshol P, Jazet IM: Immediate and long-term effects of addition of exercise to a 16-week very low calorie diet on low-grade inflammation in obese, insulin-dependent type 2 diabetic patients. Food Chem Toxicol 2011, 49(12):3104-3111.

37. Skender ML, Goodrick GK, Del Junco DJ, Reeves RS, Darnell L, Gotto AM, Foreyt JP: Comparison of 2-year weight loss trends in behavioral treatments of obesity: diet, exercise, and combination interventions. J Am Diet Assoc 1996, 96(4):342-346.

38. Racette SB, Weiss EP, Villareal DT, Arif H, Steger-May K, Schechtman KB, Fontana L, Klein S, Holloszy JO: One year of caloric restriction in humans: feasibility and effects on body composition and abdominal adipose tissue. J Gerontol A Biol Sci Med Sci 2006, 61(9):943-950.

39. Pritchard JE, Nowson CA, Wark JD: A worksite program for overweight middle-aged men achieves lesser weight loss with exercise than with dietary change. J Am Diet Assoc 1997, 97(1):37-42.

40. Anderssen SA, Hjermann I, Urdal P, Torjesen PA, Holme I: Improved carbohydrate metabolism after physical training and dietary intervention in individuals with the "atherothrombogenic syndrome'. Oslo Diet and Exercise Study (ODES). A randomized trial. J Intern Med 1996, 240(4):203-209.

41. Reseland JE, Anderssen SA, Solvoll K, Hjermann I, Urdal P, Holme I, Drevon CA: Effect of long-term changes in diet and exercise on plasma leptin concentrations. Am J Clin Nutr 2001, 73(2):240-245.

42. Andrews RC, Cooper AR, Montgomery AA, Norcross AJ, Peters TJ, Sharp DJ, Jackson N, Fitzsimons K, Bright J, Coulman K, England CY, Gorton J, McLenaghan A, Paxton E, Polet A, Thompson C, Dayan CM: Diet or diet plus physical activity versus usual care in patients with newly diagnosed type 2 diabetes: the Early ACTID randomised controlled trial. Lancet 2011, 378(9786):129-139

43. Borg P, Kukkonen-Harjula K, Fogelholm M, Pasanen M: Effects of walking or resistance training on weight loss maintenance in obese, middle-aged men: a randomized trial. Int J Obes Relat Metab Disord 2002, 26(5):676-683.

44. Brekke HK, Jansson PA, Lenner RA: Long-term (1- and 2-year) effects of lifestyle intervention in type 2 diabetes relatives. Diabetes Res Clin Pract 2005, 70(3):225-234.

45. Fogelholm M, Kukkonen-Harjula K, Nenonen A, Pasanen M: Effects of walking training on weight maintenance after a very-low-energy diet in premenopausal obese women: a randomized controlled trial. Arch Intern Med 2000, 160(14):2177-2184.

46. Foster-Schubert KE, Alfano CM, Duggan CR, Xiao L, Campbell KL, Kong A, Bain CE, Wang CY, Blackburn GL, McTiernan A: Effect of diet and exercise, alone or combined, on weight and body composition in overweight-to-obese postmenopausal women. Obesity (Silver Spring) 2012, 20(8):1628-1638.

47. Nicklas BJ, Ambrosius W, Messier SP, Miller GD, Penninx BW, Loeser RF, Palla S, Bleecker E, Pahor M: Diet-induced weight loss, exercise, and chronic inflammation in older, obese adults: a randomized controlled clinical trial. Am J Clin Nutr 2004, 79(4):544-551.

48. Pan XR, Li GW, Hu YH, Wang JX, Yang WY, An ZX, Hu ZX, Lin J, Xiao JZ, Cao HB, Liu PA, Jiang XG, Jiang $Y$, Wang JP, Zheng $H$, Zhang $H$, Bennett $P H$, Howard BV: Effects of diet and exercise in preventing NIDDM in people with impaired glucose tolerance. The Da Qing IGT and Diabetes Study. Diabetes Care 1997, 20(4):537-544.

49. Wood PD, Stefanick ML, Williams PT, Haskell WL: The effects on plasma lipoproteins of a prudent weight-reducing diet, with or without exercise, in overweight men and women. N Engl J Med 1991, 325(7):461-466.
50. Messier SP, Mihalko SL, Legault C, Miller GD, Nicklas BJ, DeVita P, Beavers DP, Hunter DJ, Lyles MF, Eckstein F, Williamson JD, Carr JJ, Guermazi A, Loeser RF: Effects of intensive diet and exercise on knee joint loads, inflammation, and clinical outcomes among overweight and obese adults with knee osteoarthritis: the IDEA randomized clinical trial. JAMA 2013, 310(12):1263-1273.

51. Messier SP, Loeser RF, Miller GD, Morgan TM, Rejeski WJ, Sevick MA, Ettinger WH Jr, Pahor M, Williamson JD: Exercise and dietary weight loss in overweight and obese older adults with knee osteoarthritis: the Arthritis, Diet, and Activity Promotion Trial. Arthritis Rheum 2004, 50(5):1501-1510.

52. Christensen P, Frederiksen R, Bliddal H, Riecke BF, Bartels EM, Henriksen M, Juul SRT, Gudbergsen H, Winther K, Astrup A, Christensen R: Comparison of three weight maintenance programs on cardiovascular risk, bone and vitamins in sedentary older adults. Obesity (Silver Spring) 2013, 21(10):1982-1990.

53. Curioni CC, Lourenco PM: Long-term weight loss after diet and exercise: a systematic review. Int J Obes (Lond) 2005, 29(10):1168-1174.

54. Franz MJ, VanWormer JJ, Crain AL, Boucher JL, Histon T, Caplan W, Bowman JD, Pronk NP: Weight-loss outcomes: a systematic review and meta-analysis of weight-loss clinical trials with a minimum 1-year follow-up. J Am Diet Assoc 2007, 107(10):1755-1767.

55. Gray LJ, Cooper N, Dunkley A, Warren FC, Ara R, Abrams K, Davies MJ, Khunti K, Sutton A: A systematic review and mixed treatment comparison of pharmacological interventions for the treatment of obesity. Obes Rev 2012, 13(6):483-498.

56. Yanovski SZ, Yanovski JA: Long-term drug treatment for obesity: a systematic and clinical review. JAMA 2014, 311(1):74-86.

57. Look ARG, Wing RR, Bolin P, Brancati FL, Bray GA, Clark JM, Coday M, Crow RS, Curtis JM, Egan CM, Espeland MA, Evans M, Foreyt JP, Ghazarian S, Gregg EW, Harrison B, Hazuda HP, Hill JO, Horton ES, Hubbard VS, Jakicic JM, Jeffery RW, Johnson KC, Kahn SE, Kitabchi AE, Knowler WC, Lewis CE, Maschak-Carey BJ, Montez MG, Murillo A, et al: Cardiovascular effects of intensive lifestyle intervention in type 2 diabetes. N Engl J Med 2013, 369(2):145-154.

58. Zhou YH, Ma XQ, Wu C, LU J, Zhang SS, Guo J, Wu SQ, Ye XF, Xu JF, He J: Effect of anti-obesity drug on cardiovascular risk factors: a systematic review and meta-analysis of randomized controlled trials. PLOS One 2012, 7(6):e39062

59. Hamman RF, Wing RR, Edelstein SL, Lachin JM, Bray GA, Delahanty L, Hoskin M, Kriska AM, Mayer-Davis EJ, Pi-Sunyer X, Regensteiner J, Venditti B, Wylie-Rosett J: Effect of weight loss with lifestyle intervention on risk of diabetes. Diabetes Care 2006, 29(9):2102-2107.

60. de Koning L, Merchant AT, Pogue J, Anand SS: Waist circumference and waist-to-hip ratio as predictors of cardiovascular events: meta-regression analysis of prospective studies. Eur Heart J 2007, 28(7):850-856.

61. Dattilo AM, Kris-Etherton PM: Effects of weight reduction on blood lipids and lipoproteins: a meta-analysis. Am J Clin Nutr 1992, 56(2):320-328.

62. Kraus WE, Houmard JA, Duscha BD, Knetzger KJ, Wharton MB, McCartney JS, Bales CW, Henes S, Samsa GP, Otvos JD, Kulkarni KR, Slentz CA: Effects of the amount and intensity of exercise on plasma lipoproteins. $N$ Engl $J$ Med 2002, 347(19):1483-1492.

63. Lee DC, Sui X, Artero EG, Lee IM, Church TS, McAuley PA, Stanford FC, Kohl HW 3rd, Blair SN: Long-term effects of changes in cardiorespiratory fitness and body mass index on all-cause and cardiovascular disease mortality in men: the Aerobics Center Longitudinal Study. Circulation 2011, 124(23):2483-2490.

64. Blair SN, Kohl HW 3rd, Paffenbarger RS Jr, Clark DG, Cooper KH, Gibbons LW: Physical fitness and all-cause mortality. A prospective study of healthy men and women. JAMA 1989, 262(17):2395-2401.

65. Kodama S, Saito K, Tanaka S, Maki M, Yachi Y, Asumi M, Sugawara A, Totsuka K, Shimano H, Ohashi Y, Yamada N, Sone H: Cardiorespiratory fitness as a quantitative predictor of all-cause mortality and cardiovascular events in healthy men and women: a meta-analysis. JAMA 2009, 301(19):2024-2035.

66. Moreno SG, Sutton AJ, Turner EH, Abrams KR, Cooper NJ, Palmer TM, Ades AE: Novel methods to deal with publication biases: secondary analysis of antidepressant trials in the FDA trial registry database and related journal publications. BMJ 2009, 339:b2981.

\section{doi:10.1186/2046-4053-3-130}

Cite this article as: Schwingshackl et al:: Impact of long-term lifestyle programmes on weight loss and cardiovascular risk factors in overweight/obese participants: a systematic review and network meta-analysis. Systematic Reviews 2014 3:130. 